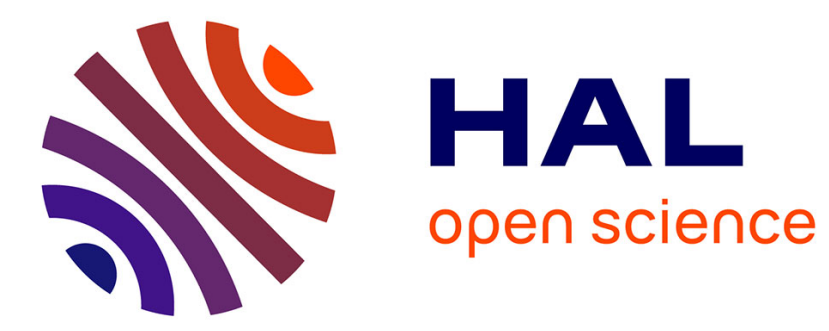

\title{
Finite element computation of Green's functions
}

Denis Duhamel

\section{To cite this version:}

Denis Duhamel. Finite element computation of Green's functions. Engineering Analysis with Boundary Elements, 2007, à paraitre. 10.1016/j.enganabound.2007.04.002 . hal-00150429

\section{HAL Id: hal-00150429 \\ https://hal.science/hal-00150429}

Submitted on 30 May 2007

HAL is a multi-disciplinary open access archive for the deposit and dissemination of scientific research documents, whether they are published or not. The documents may come from teaching and research institutions in France or abroad, or from public or private research centers.
L'archive ouverte pluridisciplinaire HAL, est destinée au dépôt et à la diffusion de documents scientifiques de niveau recherche, publiés ou non, émanant des établissements d'enseignement et de recherche français ou étrangers, des laboratoires publics ou privés. 


\title{
FINITE ELEMENT COMPUTATION OF GREEN'S FUNCTIONS
}

\author{
D. Duhamel \\ LAMI - unité mixte ENPC/LCPC, Institut Navier \\ 6 et 8 Avenue Blaise Pascal, \\ Cité Descartes, Champs sur Marne, \\ 77455 Marne la Vallée, cedex 2, France \\ Tel: 33164153728 \\ Fax: 33164153741 \\ email : duhamel@lami.enpc.fr \\ Total number of pages $=49$ \\ Total number of figures $=15$
}

30 May 2007 


\begin{abstract}
Green's functions are important mathematical tools in mechanics and in other parts of physics. For instance, the boundary element method needs to know the Green's function of the problem to compute its numerical solution. However, Green's functions are only known in a limited number of cases, often under the form of complex analytical expressions. In this article, a new method is proposed to calculate Green's functions for any linear homogeneous medium from a simple finite element model. The method relies on the theory of wave propagation in periodic media and requires the knowledge of the finite element dynamic stiffness matrix of only one period. Several examples are given to check the accuracy and the efficiency of the proposed numerical Green's function.
\end{abstract}

Key words: Green's function, periodic structure, wave, waveguide, finite element, dynamic. 


\section{Introduction}

This article presents an algorithm for the general numerical computation of Green's functions for linear homogeneous media of arbitrary types from simple finite element models. Green's functions give the solution of a problem described by a partial differential equation when it is submitted to a unit excitation. These are fundamental tools since if the Green's function for a linear differential equation is known, then it is possible to calculate, by simple convolution type integrals, the solutions of the same problem for an arbitrary loading. They are also the basic tools to formulate a boundary integral equation to solve the partial differential equation describing the physical problem. So the search for Green's functions for the various physical problems in acoustics, solid mechanics, heat conduction and other parts of mechanics and physics played an important role in the past for solving these problems.

For the simplest cases the Green's functions are known for a long time and are described in classical books like Morse and Feshbach [1], Courant and Hilbert [2], Carslaw and Jaeger [3], Roach [4] and Bonnet [5]. One has, for instance, solutions for two- and three-dimensional acoustics, for two- and threedimensional elastostatics and elastodynamics or for diffusion equations. Solutions can be in the time or frequency domains according to the equation describing the physical phenomenon. These solutions are given as analytical formulas, often involving special functions. 
However, for more complex problems, solutions are not so easy to find and the computation of the Green's function can still be an open question. If we consider only mechanical problems and recent works, we can cite DiazContreras and Nomura [6] who found the Green's function for Mindlin plates using eigenfunction expansions obtained numerically by the Galerkin method. Several authors like Dravinski and Zheng [7] and Phan et al. [8] have also considered the computation of Green's functions for non isotropic elasticity. As the expression for this function is complex, Sales and Gray [9] and Tonon et al. [10] developed efficient methods for calculating the general 3D Green's function for anisotropic media.

Other authors searched for Green's functions related to multilayer structures. For example, Yang and Pan [11] found the static Green's function for anisotropic trimaterials while Bai et al. [12] considered a layered isotropic plate and found the elastodynamic Green's function by using a modal summation. The Green's functions for half spaces were also studied by many authors like Pan [13 who found the Green's functions for static multilayered transversely isotropic half spaces and Spies [14 who considered the general static anisotropic Green's function for a half-space. He and Lim [15 studied the Green's function for a soft half-space with surface stress. More complicated cases with several behaviors were also considered by Ding et al. [16] where the authors found the Green's function for transversally isotropic piezoelectric materials. In all these cases except the simplest ones, the Green's functions are found by complex 
analytical expressions, often involving series expansions. For media with complex properties involving several materials or coupled mechanical models, the Green's functions are generally unknown.

Here, we present a numerical method to find such Green's functions. It is supposed that the medium is two-dimensional, linear, infinite, homogeneous or periodic but can otherwise be arbitrary. The approach is based on the theory of periodic media and one supposes that a finite element model of a period is given from standard finite element software. Similar ideas were used in Mace et al. [17] and Duhamel et al. [18] in the one-dimensional case. The twodimensional case was considered by Langley [19,20,21] who found expressions based on Bloch's waves for the response to point loading for harmonic and impulsive loads. But the analysis was mainly theoretical with simple examples and no connection with the finite element method was made. We consider here only two-dimensional problems to simplify. From the dynamic stiffness matrix, waves in different directions are calculated and the Green's function is found as an integral of wave functions.

The paper is divided in two parts. In the first part, the numerical method is presented and the formula giving the Green's function is obtained. In the second part, different examples are given to check the method against known analytical Green's functions and a more complex example is also presented to calculate the Green's function of a two materials periodic medium for which no simple solutions are known. 


\section{Green's functions calculation}

We consider an infinite periodic or homogeneous medium. The Green's function $G\left(\mathbf{x}_{\mathbf{1}}, \mathbf{x}_{\mathbf{2}}\right)$ is the response at a point $\mathbf{x}_{\mathbf{1}}$ in the medium for a unitary excitation at an other point $\mathbf{x}_{\mathbf{2}}$ as in figure 1. As an homogeneous medium is only a special case of a periodic medium, in the following, only periodic media will be considered. The analysis is also limited to the two-dimensional case and the elementary period is defined by the domain $0 \leq x_{1} \leq L_{1}$ and $0 \leq x_{2} \leq L_{2}$. The excitation is in a cell and the point where the response is calculated can be in another cell, see figure 2.

\subsection{Pseudo periodic functions}

It is proved in appendix A that a function $U\left(x_{1}, x_{2}\right)$ defined on the twodimensional periodic medium can be decomposed as an integral of pseudo periodic functions

$$
U\left(x_{1}, x_{2}\right)=\int_{-\frac{\pi}{L_{2}}}^{\frac{\pi}{L_{2}}} e^{i k x_{2}} \hat{U}\left(x_{1}, k, x_{2}\right) d k
$$

where $\hat{U}\left(x_{1}, k, x_{2}\right)$ is a periodic function in $x_{2}$ with period $L_{2}$. Along direction 1, we use a decomposition in Bloch waves as it is usual in periodic media. Finally, the general solution can be obtained from functions $u\left(x_{1}, k, x_{2}\right)=$

$e^{i k x_{2}} \hat{U}\left(x_{1}, k, x_{2}\right)$ with the following pseudo periodic properties 


$$
\begin{aligned}
& u\left(x_{1}, k, x_{2}+m_{2} L_{2}\right)=e^{i k m_{2} L_{2}} u\left(x_{1}, k, x_{2}\right) \\
& u\left(x_{1}+m_{1} L_{1}, k, x_{2}\right)=e^{i m_{1} \mu_{1}} u\left(x_{1}, k, x_{2}\right)
\end{aligned}
$$

where $m_{1}$ and $m_{2}$ are integers and the number $k$ is real and belongs to $\left[-\frac{\pi}{L_{2}}, \frac{\pi}{L_{2}}\right]$. Along dimension 1 we will suppose, according to the Floquet's theorem, that $\mu_{1}$ is a complex number. In the following, we are looking for numerical approximations of functions which satisfy relations (2)).

\subsection{Behavior of a cell}

A cell is defined as a two-dimensional domain from which the whole plane can be obtained by tessellation. It is the smallest period of the medium and was defined previously as the domain $0 \leq x_{1} \leq L_{1}$ and $0 \leq x_{2} \leq L_{2}$. A cell can be meshed with an arbitrary number of elements using the full possibilities of usual finite element software. The discrete dynamic behavior of a cell obtained from a finite element model at a frequency $\omega$ is given by

$$
\left(\mathbf{K}-i \omega \mathbf{C}-\omega^{2} \mathbf{M}\right) \mathbf{q}=\mathbf{f}
$$

where $\mathbf{K}, \mathbf{M}$ and $\mathbf{C}$ are the stiffness, mass and damping matrices respectively, $\mathbf{f}$ is the loading vector and $\mathbf{q}$ the vector of the degrees of freedom. The stiffness, mass and damping matrices could be obtained from any commercial finite element software and so this allows the consideration of cells with complex structures. The size of these matrices depends on the number of elements used to mesh the cell and can be arbitrarily increased for a better precision 
of the results. Introducing the dynamic stiffness matrix $\tilde{\mathbf{D}}=\mathbf{K}-i \omega \mathbf{C}-$ $\omega^{2} \mathbf{M}$, decomposing into boundary (B) and interior (I) degrees of freedom, and assuming that there are no external forces on the interior nodes, results in the following relation

$$
\left[\begin{array}{cc}
\tilde{\mathbf{D}}_{B B} & \tilde{\mathbf{D}}_{B I} \\
\tilde{\mathbf{D}}_{I B} & \tilde{\mathbf{D}}_{I I}
\end{array}\right]\left[\begin{array}{l}
\mathbf{q}_{B} \\
\mathbf{q}_{I}
\end{array}\right]=\left[\begin{array}{l}
\mathbf{f}_{B} \\
\mathbf{0}
\end{array}\right]
$$

The assumption that there are no forces on the interior degrees of freedom is satisfied for free waves inside the structure for which the forces on a cell are only produced by boundary forces from the adjacent cells. The interior degrees of freedom can thus be eliminated to get

$$
\mathbf{f}_{B}=\left(\tilde{\mathbf{D}}_{B B}-\tilde{\mathbf{D}}_{B I} \tilde{\mathbf{D}}_{I I}^{-1} \tilde{\mathbf{D}}_{I B}\right) \mathbf{q}_{B}
$$

Dropping the $B$ index, this relation is written in the sequel as

$$
\mathbf{f}=\mathbf{D q}
$$

and only boundary degrees of freedom will be considered now.

\subsection{Elimination of transverse dofs}

Consider the situation described in figure 3. The boundary degrees of freedom are divided into the longitudinal dofs, denoted $\mathbf{q}_{l}$ which are the dofs such that 
the first coordinate equals an integer number of the first period $L_{1}$ and the transverse dofs which are the other boundary dofs. These transverse dofs can be divided into two subgroups denoted $\mathbf{q}_{B}$ for the bottom dofs and $\mathbf{q}_{T}$ for the top dofs. The top dofs are obtained from the bottom dofs by the pseudo periodic boundary conditions (2).

The cells are supposed to be meshed with an equal number of nodes on their opposite edges. The boundary can be decomposed into left (L), right (R), bottom (B) and top (T) parts and the associated corners (LB), (RB), (LT) and (RT) as shown in figure 4 and in Abdel-Rahman [22]. The behavior in direction 2 is supposed to be defined by the propagation constant $e^{i k L_{2}}$ where $k$ is a wavenumber in $\left[-\frac{\pi}{L_{2}}, \frac{\pi}{L_{2}}\right]$. In this case, one has

$$
\mathbf{q}_{l}=\left[\begin{array}{c}
\mathbf{q}_{L} \\
\mathbf{q}_{R} \\
\mathbf{q}_{L B} \\
\mathbf{q}_{R B} \\
\mathbf{q}_{R T} \\
\mathbf{q}_{L T}
\end{array}\right]
$$

The relations between the transverse dofs are therefore 


$$
\begin{aligned}
\mathbf{q}_{T} & =e^{i k L_{2}} \mathbf{q}_{B} \\
\mathbf{f}_{B}+e^{-i k L_{2}} \mathbf{f}_{T} & =0
\end{aligned}
$$

The behavior of a cell, given by relation (6), can also be written as

$$
\left[\begin{array}{ccc}
\mathbf{D}_{l l} & \mathbf{D}_{l B} & \mathbf{D}_{l T} \\
\mathbf{D}_{B l} & \mathbf{D}_{B B} & \mathbf{D}_{B T} \\
\mathbf{D}_{T l} & \mathbf{D}_{T B} & \mathbf{D}_{T T}
\end{array}\right]\left[\begin{array}{l}
\mathbf{q}_{l} \\
\mathbf{q}_{B} \\
\mathbf{q}_{T}
\end{array}\right]=\left[\begin{array}{c}
\mathbf{f}_{l} \\
\mathbf{f}_{B} \\
\mathbf{f}_{T}
\end{array}\right]
$$

One gets from (8) and rows 2 and 3 of relations (9)

$$
\left(\mathbf{D}_{B l}+e^{-i k L_{2}} \mathbf{D}_{T l}\right) \mathbf{q}_{l}+\left(\mathbf{D}_{B B}+\mathbf{D}_{T T}+e^{-i k L_{2}} \mathbf{D}_{T B}+e^{i k L_{2}} \mathbf{D}_{B T}\right) \mathbf{q}_{B}=0(10)
$$

SO

$$
\mathbf{q}_{B}=-\left(\mathbf{D}_{B B}+\mathbf{D}_{T T}+e^{-i k L_{2}} \mathbf{D}_{T B}+e^{i k L_{2}} \mathbf{D}_{B T}\right)^{-1}\left(\mathbf{D}_{B l}+e^{-i k L_{2}} \mathbf{D}_{T l}\right) \mathbf{q}_{l}(11)
$$

and finally the condensed behavior of the cell with only the longitudinal dofs is given by

$$
\begin{aligned}
\mathbf{f}_{l}= & {\left[\mathbf{D}_{l l}-\left(\mathbf{D}_{l B}+e^{i k L_{2}} \mathbf{D}_{l T}\right)\left(\mathbf{D}_{B B}+\mathbf{D}_{T T}+e^{-i k L_{2}} \mathbf{D}_{T B}+e^{i k L_{2}} \mathbf{D}_{B T}\right)^{-1}\right.} \\
& \left.\times\left(\mathbf{D}_{B l}+e^{-i k L_{2}} \mathbf{D}_{T l}\right)\right] \mathbf{q}_{l}
\end{aligned}
$$

whose notation will be simplified to

$$
\mathbf{f}_{l}=\mathbf{D}_{l} \mathbf{q}_{l}
$$


One can notice that we have

$$
{ }^{T} \mathbf{D}_{l}(k)=\mathbf{D}_{l}(-k)
$$

\subsection{Elementary degrees of freedom}

We go on by a further partitioning of the left and right boundaries. The longitudinal displacement and load vectors can be partitioned as in relation (7)

$$
\mathbf{q}_{l}=\left[\begin{array}{c}
\mathbf{q}_{L} \\
\mathbf{q}_{R} \\
\mathbf{q}_{L B} \\
\mathbf{q}_{R B} \\
\mathbf{q}_{R T} \\
\mathbf{q}_{L T}
\end{array}\right] \mathbf{f}_{l}=\left[\begin{array}{c}
\mathbf{f}_{L} \\
\mathbf{f}_{R} \\
\mathbf{f}_{L B} \\
\mathbf{f}_{R B} \\
\mathbf{f}_{R T} \\
\mathbf{f}_{L T}
\end{array}\right]
$$

So, the pseudo periodic conditions (2) lead to the following relations between the displacement degrees of freedom

$$
\begin{aligned}
\mathbf{q}_{R} & =e^{i \mu_{1}} \mathbf{q}_{L} \\
\mathbf{q}_{R B} & =e^{i \mu_{1}} \mathbf{q}_{L B} \\
\mathbf{q}_{R T} & =e^{i\left(\mu_{1}+k L_{2}\right)} \mathbf{q}_{L B} \\
\mathbf{q}_{L T} & =e^{i k L_{2}} \mathbf{q}_{L B}
\end{aligned}
$$


We see that the displacement components depend on an elementary set of degrees of freedom which is denoted $\mathbf{q}_{r}^{T}=\left[\begin{array}{ll}\mathbf{q}_{L} & \mathbf{q}_{L B}\end{array}\right]^{T}$ and is presented in figure 4. The vector $\mathbf{q}_{l}$ can then be expressed as

$$
\mathbf{q}_{l}=\left(\mathbf{W}_{0}+e^{i \mu_{1}} \mathbf{W}_{1}\right) \mathbf{q}_{r}
$$

where the matrices $\mathbf{W}_{0}$ and $\mathbf{W}_{1}$ depend on the wave number $k$ and are given by the following relations

$$
\mathbf{W}_{0}=\left[\begin{array}{ll}
\mathbf{I} & \mathbf{O} \\
\mathbf{O} & \mathbf{O} \\
\mathbf{O} & \mathbf{I} \\
\mathbf{O} & \mathbf{O} \\
\mathbf{O} & \mathbf{O} \\
\mathbf{O} & e^{i k L_{2}} \mathbf{I}
\end{array}\right] \quad \mathbf{W}_{1}=\left[\begin{array}{ll}
\mathbf{O} & \mathbf{O} \\
\mathbf{I} & \mathbf{O} \\
\mathbf{O} & \mathbf{O} \\
\mathbf{O} & \mathbf{I} \\
\mathbf{O} & e^{i k L_{2}} \mathbf{I} \\
\mathbf{O} & \mathbf{O}
\end{array}\right]
$$

\subsection{Spectral problem}

The equilibrium conditions between adjacent cells written on the reduced set of degrees of freedom lead to the following relations between the components of the force vector

$$
e^{i \mu_{1}} \mathbf{f}_{L}+\mathbf{f}_{R}=0
$$




$$
e^{i \mu_{1}} \mathbf{f}_{L B}+\mathbf{f}_{R B}+e^{i\left(\mu_{1}-k L_{2}\right)} \mathbf{f}_{L T}+e^{-i k L_{2}} \mathbf{f}_{R T}=0
$$

that can also be written as

$$
\left[\begin{array}{cccccc}
e^{i \mu_{1}} \mathbf{I} & \mathbf{I} & \mathbf{O} & \mathbf{O} & \mathbf{O} & \mathbf{O} \\
\mathbf{O} & \mathbf{O} & e^{i \mu_{1}} \mathbf{I} & \mathbf{I} & e^{-i k L_{2}} \mathbf{I} & e^{i \mu_{1}-i k L_{2}} \mathbf{I}
\end{array}\right] \mathbf{f}_{l}=0
$$

or

$$
\left(e^{i \mu_{1}} \mathbf{W}_{0}^{*}+\mathbf{W}_{1}^{*}\right) \mathbf{f}_{l}=0
$$

Relations (13), (17) and (21) finally give

$$
\left(e^{i \mu_{1}} \mathbf{W}_{0}^{*}+\mathbf{W}_{1}^{*}\right) \mathbf{D}_{l}\left(\mathbf{W}_{0}+e^{i \mu_{1}} \mathbf{W}_{1}\right) \mathbf{q}_{r}=0
$$

that can also be written as

$$
\left[e^{i \mu_{1}}\left(\mathbf{W}_{0}^{*} \mathbf{D}_{l} \mathbf{W}_{0}+\mathbf{W}_{1}^{*} \mathbf{D}_{l} \mathbf{W}_{1}\right)+e^{2 i \mu_{1}} \mathbf{W}_{0}^{*} \mathbf{D}_{l} \mathbf{W}_{1}+\mathbf{W}_{1}^{*} \mathbf{D}_{l} \mathbf{W}_{0}\right] \mathbf{q}_{r}=0
$$

or

$$
\left(\mathbf{A}_{0}+e^{i \mu_{1}} \mathbf{A}_{1}+e^{2 i \mu_{1}} \mathbf{A}_{2}\right) \mathbf{q}_{r}=0
$$

where

$$
\begin{aligned}
& \mathbf{A}_{0}=\mathbf{W}_{1}^{*} \mathbf{D}_{l} \mathbf{W}_{0} \\
& \mathbf{A}_{1}=\mathbf{W}_{0}^{*} \mathbf{D}_{l} \mathbf{W}_{0}+\mathbf{W}_{1}^{*} \mathbf{D}_{l} \mathbf{W}_{1} \\
& \mathbf{A}_{2}=\mathbf{W}_{0}^{*} \mathbf{D}_{l} \mathbf{W}_{1}
\end{aligned}
$$


Relation (24) give the spectral problem to be solved to find the propagation constants $e^{i \mu_{1}}$ and the vectors $\mathbf{q}_{r}$. The propagation constant $e^{i \mu_{1}}$, the matrices $\mathbf{A}_{0}, \mathbf{A}_{1}, \mathbf{A}_{2}$ and the eigenvector $\mathbf{q}_{r}$ depend on the transverse wavenumber $k$ but to simplify the notation this dependence is not explicitly written. The eigenvector of (24) associated with the eigenvalue $e^{i \mu_{i}}$ is denoted by $\mathbf{q}_{i}$. By taking the determinant of (23) and of its transpose for $-k$ and noting that $\mathbf{W}_{i}^{*}={ }^{t} \overline{\mathbf{W}}_{i}={ }^{t} \mathbf{W}_{i}(-k)$, using relation (14), it can be shown that if $e^{i \mu_{i}}$ is an eigenvalue for the wavenumber $k$ then $e^{-i \mu_{i}}$ is also an eigenvalue for the wavenumber $-k$. These represent a pair of positive and negative-going waves. This is true for any shape or property of the cell.

The $2 n$ eigenvalues of equation (24) can be split into two sets of $n^{+}$and $n^{-}$eigenvalues and eigenvectors with $2 n=n^{+}+n^{-}$. They are denoted by $\left(e^{i \mu_{i}^{+}}, \mathbf{q}_{i}^{+}\right)$and $\left(e^{i \mu_{i}^{-}}, \mathbf{q}_{i}^{-}\right)$, with the first set such that $\left|e^{i \mu_{i}^{+}}\right| \leq 1$. From an eigenvector $\mathbf{q}_{i}$, one gets the longitudinal vector by relation (17) and the longitudinal component of the force vector applied on a cell is given by

$$
\mathbf{f}_{i}=\mathbf{D}_{l}\left(\mathbf{W}_{0}+e^{i \mu_{i}} \mathbf{W}_{1}\right) \mathbf{q}_{i}
$$

The forces of left cells on right cells are then given by

$$
\mathbf{f}_{i}^{r}=\mathbf{W}_{0}^{*} \mathbf{f}_{i}=\mathbf{W}_{0}^{*} \mathbf{D}_{l}\left(\mathbf{W}_{0}+e^{i \mu_{i}} \mathbf{W}_{1}\right) \mathbf{q}_{i}
$$

In the case $\left|e^{i \mu_{i}}\right|=1$, the set of positive-going waves must contain the waves which are such that $\operatorname{Re}\left\{i \omega \mathbf{q}_{i}^{H} \mathbf{f}_{i}^{r}\right\}>0$ where $\mathbf{f}_{i}^{r}$ is the reduced set of force 
degrees of freedom on the boundary obtained by relation (27). The eigenvalue $e^{i \mu_{i}^{-}}$, in the second set, is associated with waves such that $\operatorname{Re}\left\{i \omega \mathbf{q}_{i}^{H} \mathbf{f}_{i}^{r}\right\}<0$.

\subsection{Green's function on a periodic array}

We can now build the complete Green's function. The Green's function gives the solution of the problem defined on the periodic medium for a point force excitation. Suppose that the force is located at point $(0,0)$, we look for a Green's function which is such that in the domain $m_{1} \geq 0$ the solution is given by

$$
\mathbf{q}_{r}\left(m_{1} L_{1}, m_{2} L_{2}\right)=\int_{-\frac{\pi}{L_{2}}}^{\frac{\pi}{L_{2}}} \sum_{i=1}^{i=n^{+}} a_{i}^{+} \mathbf{q}_{i}^{+} e^{i m_{1} \mu_{i}^{+}} e^{i k m_{2} L_{2}} d k
$$

because the waves must propagate from the source to infinity, so the negative waves do not appear in the sum. In the domain $m_{1} \leq 0$, the solution must be

$$
\mathbf{q}_{r}\left(m_{1} L_{1}, m_{2} L_{2}\right)=\int_{-\frac{\pi}{L_{2}}}^{\frac{\pi}{L_{2}}} \sum_{i=1}^{i=n^{-}} a_{i}^{-} \mathbf{q}_{i}^{-} e^{i m_{1} \mu_{i}^{-}} e^{i k m_{2} L_{2}} d k
$$

At $m_{1}=0$ there are continuity of the displacements and the equilibrium condition on the forces can be written by analogy with formula (21) by

$$
\sum_{i=1}^{i=n^{+}} \mathbf{W}_{0}^{*} \mathbf{f}_{i}^{+}+\sum_{i=1}^{i=n^{-}} e^{-i \mu_{i}^{-}} \mathbf{W}_{1}^{*} \mathbf{f}_{i}^{-}=-\mathbf{f}_{e x t}^{r}
$$

where $\mathbf{f}_{i}^{-}$and $\mathbf{f}_{i}^{+}$are, the respective contributions of the negative and positive waves on the forces. The expression of $\mathbf{f}_{e x t}$ for a point force excitation of 
amplitude $\mathbf{F}$ is given in appendix $\mathrm{A}$. The relations on the displacements and forces lead for each $k$ to

$$
\begin{gathered}
\sum_{i=1}^{i=n^{+}} a_{i}^{+} \mathbf{q}_{i}^{+}-\sum_{i=1}^{i=n^{-}} a_{i}^{-} \mathbf{q}_{i}^{-}=0 \\
\sum_{i=1}^{i=n^{+}} a_{i}^{+} \mathbf{W}_{0}^{*} \mathbf{D}_{l}\left(\mathbf{W}_{0}+e^{i \mu_{i}^{+}} \mathbf{W}_{1}\right) \mathbf{q}_{i}^{+} \\
+\sum_{i=1}^{i=n^{-}} a_{i}^{-} \mathbf{W}_{1}^{*} \mathbf{D}_{l}\left(e^{-i \mu_{i}^{-}} \mathbf{W}_{0}+\mathbf{W}_{1}\right) \mathbf{q}_{i}^{-}=-\frac{L_{2}}{2 \pi} \mathbf{F}
\end{gathered}
$$

We get $2 n$ relations for $2 n$ unknowns. So these relations allow to get the amplitudes $a_{i}^{+}$and $a_{i}^{-}$and then the values of the Green's function from relations (28) and (29). So the Green's function has been found as an integral of solutions to one-dimensional periodic problems.

\subsection{Numerical calculation of integrals}

The Green's function is obtained from relations (28) and (29). These relations are of the following form

$$
\mathbf{q}_{r}\left(m_{1} L_{1}, m_{2} L_{2}\right)=\int_{-\frac{\pi}{L_{2}}}^{\frac{\pi}{L_{2}}} \mathbf{Q}(k) e^{i k m_{2} L_{2}} d k
$$

with a function $\mathbf{Q}(k)$ which can be singular. Considerations involving the continuous case suggest that the singularities are of type $1 / \sqrt{K^{2}-k^{2}}$ for some $\mathrm{K}$. To allow a correct evaluation of the integral it is necessary to improve the numerical quadrature. In a first step we look for the singular points in the interval $\left[-\frac{\pi}{L_{2}}, \frac{\pi}{L_{2}}\right]$ by finding the maxima of the function $|\mathbf{Q}(k)|$. Denoting 
$k_{i}, i=1 \ldots n_{s}$ these singular points, the integrals are calculated by changing the variable as in

$$
\int_{a}^{k_{i}} \mathbf{Q}(k) e^{i k m_{2} L_{2}} d k=\int_{0}^{\sqrt{k_{i}^{2}-a^{2}}} \frac{u}{\sqrt{k_{i}^{2}-u^{2}}} \mathbf{Q}\left(\sqrt{k_{i}^{2}-u^{2}}\right) e^{i \sqrt{k_{i}^{2}-u^{2}} m_{2} L_{2}} d k
$$

which allows to remove singularities in $1 / \sqrt{k_{i}^{2}-k^{2}}$ in Q. Similar expressions are used for the singularities at lower interval bounds and for negative values of $\mathrm{k}$.

At the present state no further optimization was made to improve the numerical evaluation of these integrals. For the following examples the computing time can be evaluated to a few seconds per couple of source and receiver points for each frequency. The program is written with MATLAB ${ }^{\circledR}$ and some improvement could be expected by considering the same computation in $\mathrm{C}$ or Fortran. One should also see from formulas (28) and (29) that the computation for one or several points in space are quite similar. Most of the computing time is devoted to finding the wavefunctions and the eigenvalues which need to be done only once. After it is easy to compute the Green's functions for different points sources and different receivers with little additional cost. One should also notice that formula (28) requires the computation of oscillatory integrals and for points far enough from the sources asymptotic formulas as the stationary phase method could be used to dramatically reduce the computing time. All these issues should be discussed in more details if the present method is used in a BEM problem. 


\subsection{Generalization to non rectangular cells}

In the precedent discussion the elementary cell was supposed to be rectangular. Consider now a quadrilateral cell as in figure 5 which has a diamond shape. Along direction 2 the pseudo periodic condition does not change. Along

direction 1 one has to substitute $e^{i\left(\mu_{1}+k d\right)}$ to $e^{i \mu_{1}}$ in the precedent relations. For example relation (24) should be substituted by

$$
\left(\mathbf{A}_{0}+e^{i \mu_{1}}\left(e^{i k d} \mathbf{A}_{1}\right)+e^{2 i \mu_{1}}\left(e^{2 i k d} \mathbf{A}_{2}\right)\right) \mathbf{q}_{r}=0
$$

One has also to pay attention that $m_{1}$ steps along direction 1 and $m_{2}$ steps along direction 2 from the origin lead to the point $\left(m_{1} L 1, m_{1} d+m_{2} L 2\right)$.

\section{$3 \quad$ Examples}

\subsection{Acoustics}

The Green's function of the two-dimensional acoustics is the radiating solution of

$$
\Delta p+K^{2} p=-\delta(r)
$$


where $K=\omega / c$ is the wavenumber and $c$ the sound velocity. It is well known that the solution of this equation for the time dependence $e^{-i \omega t}$ is given by

$$
G(r)=\frac{i}{4} H_{0}(K r)
$$

where $H_{0}$ is the Hankel function of order zero and first type. We first propose to check the numerical procedure developed in this article with this Green's function. Consider the rectangular four nodes acoustic element of size $a \times b$ presented in figure 6, the stiffness and mass matrices are given by

$$
\mathbf{K}=\left[\begin{array}{cccc}
\frac{1}{3} \frac{b^{2}+a^{2}}{a b} & \frac{1}{6} \frac{-2 b^{2}+a^{2}}{a b} & -\frac{1}{6} \frac{b^{2}+a^{2}}{a b} & -\frac{1}{6} \frac{-b^{2}+2 a^{2}}{a b} \\
\frac{1}{6} \frac{-2 b^{2}+a^{2}}{a b} & \frac{1}{3} \frac{b^{2}+a^{2}}{a b} & -\frac{1}{6} \frac{-b^{2}+2 a^{2}}{a b} & -\frac{1}{6} \frac{b^{2}+a^{2}}{a b} \\
-\frac{1}{6} \frac{b^{2}+a^{2}}{a b} & -\frac{1}{6} \frac{-b^{2}+2 a^{2}}{a b} & \frac{1}{3} \frac{b^{2}+a^{2}}{a b} & \frac{1}{6} \frac{-2 b^{2}+a^{2}}{a b} \\
-\frac{1}{6} \frac{-b^{2}+2 a^{2}}{a b} & -\frac{1}{6} \frac{b^{2}+a^{2}}{a b} & \frac{1}{6} \frac{-2 b^{2}+a^{2}}{a b} & \frac{1}{3} \frac{b^{2}+a^{2}}{a b} \\
\mathbf{M}=\frac{a b}{4 c^{2}} \\
\frac{4}{9} \frac{2}{9} \frac{1}{9} \frac{2}{9} \frac{2}{9} \frac{1}{9} \frac{1}{9} \frac{2}{9} \frac{2}{9} \frac{4}{9}
\end{array}\right]
$$


and then the dynamic stiffness matrix is $\mathbf{D}=\mathbf{K}-\omega^{2} \mathbf{M}$. As in this case there is only the $\mathbf{q}_{L B}$ degree of freedom, the matrices $\mathbf{W}_{0}$ and $\mathbf{W}_{1}$ are given by

$$
\mathbf{W}_{0}=\left[\begin{array}{c}
1 \\
0 \\
0 \\
e^{i k b}
\end{array}\right] \quad \mathbf{W}_{1}=\left[\begin{array}{c}
0 \\
1 \\
e^{i k b} \\
\\
0
\end{array}\right]
$$

Using relations (24) the matrices of the spectral problem, which in this case reduce to simple scalars, are given by

$$
\begin{aligned}
A_{0}(k) & =\mathbf{W}_{1}^{*}(k) \mathbf{D}_{l}(k) \mathbf{W}_{0}(k) \\
& =-\frac{1}{18}\left[12 \frac{b}{a}-6 \frac{a}{b}+2 k^{2} a b+\left(6 \frac{b}{a}+6 \frac{a}{b}+k^{2} a b\right) \cos (k b)\right] \\
A_{1}(k) & =\mathbf{W}_{0}^{*}(k) \mathbf{D}_{l}(k) \mathbf{W}_{0}(k)+\mathbf{W}_{1}^{*}(k) \mathbf{D}_{l}(k) \mathbf{W}_{1}(k) \\
& =-\frac{2}{9}\left[-6 \frac{b}{a}-6 \frac{a}{b}+2 k^{2} a b+\left(-3 \frac{b}{a}+6 \frac{a}{b}+k^{2} a b\right) \cos (k b)\right] \\
A_{2}(k) & =\mathbf{W}_{0}^{*}(k) \mathbf{D}_{l}(k) \mathbf{W}_{1}(k) \\
& =-\frac{1}{18}\left[12 \frac{b}{a}-6 \frac{a}{b}+2 k^{2} a b+\left(6 \frac{b}{a}+6 \frac{a}{b}+k^{2} a b\right) \cos (k b)\right]
\end{aligned}
$$

and the solutions to the spectral problem (24) are

$$
\begin{aligned}
e^{i \mu_{1}} & =\left(-A_{1} \pm \sqrt{A_{1}^{2}-4 A_{0} A_{2}}\right) /\left(2 A_{2}\right) \\
e^{-i \mu_{1}} & =\left(-A_{1} \mp \sqrt{A_{1}^{2}-4 A_{0} A_{2}}\right) /\left(2 A_{2}\right)
\end{aligned}
$$

The signs are selected according to the rules defined in section 2.4. Choosing the components of the displacement to one, the force components are given 
by relations (30) and (31)

$$
\begin{aligned}
& F^{+}(k)=\mathbf{W}_{0}^{*}(k) f^{+}(k)=\mathbf{W}_{0}^{*}(k) \mathbf{D}_{l}(k)\left(\mathbf{W}_{0}(k)+e^{i \mu_{1}} \mathbf{W}_{1}(k)\right) \\
& F^{-}(k)=e^{i \mu_{1}} \mathbf{W}_{1}^{*}(k) f^{-}(k)=\mathbf{W}_{1}^{*}(k) \mathbf{D}_{l}(k)\left(e^{i \mu_{1}} \mathbf{W}_{0}(k)+\mathbf{W}_{1}(k)\right)
\end{aligned}
$$

and the system (31) to solve is

$$
\left[\begin{array}{cc}
1 & -1 \\
F^{+}(k) & F^{-}(k)
\end{array}\right]\left[\begin{array}{c}
a^{+} \\
a^{-}
\end{array}\right]=\left[\begin{array}{c}
0 \\
b /(2 \pi)
\end{array}\right]
$$

which yields

$$
a^{+}=a^{-}=\frac{b}{2 \pi\left(F^{+}(k)+F^{-}(k)\right)}
$$

and so in this case the Green's function is given by using formulas (28) and (29) which leads to

$$
G\left(m_{1} a, m_{2} b\right)= \begin{cases}\frac{b}{2 \pi} \int_{-\pi / b}^{\pi / b} \frac{e^{i\left(m_{1} \mu_{1}+m_{2} k b\right)}}{F^{+}(k)+F^{-}(k)} d k \quad \text { if } \quad m_{1} \geq 0 \\ \frac{b}{2 \pi} \int_{-\pi / b}^{\pi / b} \frac{e^{i\left(-m_{1} \mu_{1}+m_{2} k b\right)}}{F^{+}(k)+F^{-}(k)} d k \quad \text { if } \quad m_{1} \leq 0\end{cases}
$$

A more detailed computation shows that

$$
\begin{aligned}
F^{+}(k)+ & F^{-}(k)=-\frac{1}{9 b} \sqrt{3\left(\left(6+(K b)^{2}\right) \cos (k b)-6+2(K b)^{2}\right)} \\
& \times \sqrt{\left(-12 b^{2}+6 a^{2}+(K a b)^{2}\right) \cos (k b)-6 a^{2}-24 b^{2}+2(K a b)^{2}}
\end{aligned}
$$

For the cases $K b<<1, K a<<1, k b<<1$, one has the approximation

$$
F^{+}(k)+F^{-}(k) \approx-2 \sqrt{(k b)^{2}-(K b)^{2}}
$$


which shows that the singularity in the integral is of square root type as expected.

An example of result is presented in figure 7. The acoustic element has the dimensions $a=0.01 \mathrm{~m}$ and $b=0.01 \mathrm{~m}$. The point force excitation is supposed to be at the origin, which is also a corner of a cell, and the analysis is done for a point at $x=0.5 \mathrm{~m}$ and $y=1 \mathrm{~m}$. The sound velocity is $c=340 \mathrm{~m} / \mathrm{s}$ and the calculation is done over the frequency band $[0,1000 H z]$. One can see that the numerical Green's function is in full agreement with the analytical solution. To have a better estimate of the relative error in the computation, this error is plotted versus the number of elements used to mesh the periodic cell in figure 8. These elements are generated by ANSYS ${ }^{\circledR}$ 23] and then are read and post-processed by MATLAB ${ }^{\circledR}$ [24] to get the results. The relative error is defined by $e=\left|G_{\text {num }}-G_{\text {ana }}\right| /\left|G_{\text {ana }}\right|$ where $G_{\text {num }}$ is the numerical Green's function obtained by the present method and $G_{\text {ana }}$ is get by formula (36). In every case the cell has the dimensions $a=0.01 \mathrm{~m}$ and $b=0.01 \mathrm{~m}$ but it is meshed with $1 \times 1,2 \times 2$ or $4 \times 4$ linear elements. It is clear in figure 8 that increasing the number of elements leads to a decrease in the computational error. Another possibility is to reduce the size of the periodic cell, which can be done here since the medium is homogeneous. This is presented in figure 9 where the error is plotted versus the size of the cell. In every case only one element with $a=b$ is used for the mesh. The results show clearly that reducing the size of the cell leads to much better results. However reducing too much 
can create instabilities for low frequencies as can be seen in the curve for the size $0.0005 \mathrm{~m}$. It seems reasonable to have cells with size such that $K a>0.001$. For large frequencies the mesh must satisfy the usual requirement of having a sufficient number of nodes (5 to 10) per wavelength otherwise the displacement could not be accurately represented on a cell.

Other computations were also done for diamond shape cells as in figure 5 . Taking into account the modifications described in section 2.8 and computing the same example as for figure 7 lead to the same curves which are not reproduced here. So the proposed method is not strictly limited to rectangular cells and can be generalized to more complex shapes with appropriate changes in the equations.

\subsection{Two-dimensional elastodynamics}

An example similar to the precedent is calculated for the two-dimensional elastodynamic case for plane strain problems. The periodic cell is first taken as the square of size $0.01 \mathrm{~m} \times 0.01 \mathrm{~m}$. The material is steel with $E=2.10^{11} \mathrm{~Pa}$, $\nu=0.3$ and $\rho=7800 \mathrm{~kg} / \mathrm{m}^{3}$.

In figure 10 the analytical solution is compared with the present numerical solution for the point $(0.5 \mathrm{~m}, 0.2 \mathrm{~m})$ and a unit force at origin in the $x$ direction. The curves show the real and imaginary parts of the $x$ component of the displacement. A very good agreement between the two curves is observed. In 
figure 11 the error between the analytical and numerical solutions are plotted versus the frequency for a periodic cell made of different number of elements. The global size of the periodic cell is still $0.01 \mathrm{~m} \times 0.01 \mathrm{~m}$ but it can be divided in $1 \times 1,2 \times 2$ or $4 \times 4$ elements of degree one or in $1 \times 1$ element of degree two. It can be clearly seen that a denser mesh leads to lower errors and that using an element of degree two considerably improves the result.

In figure 12 the error is plotted versus the size of the periodic cell. Only one element of degree one per cell is used for these examples. It can be seen that the error decreases as the size of the cell is smaller. So it seems interesting to take cells with rather small sizes. These results are quite similar to the acoustic case.

Finally, figure 13 presents the numerical and analytical solutions for the frequency $1000 \mathrm{~Hz}$ along the direction $x$ for a distance varying between $0.01 \mathrm{~m}$ (the size of a cell) and $1 \mathrm{~m}$. It can be observed that the agreement is very good for all distances. So the numerical computation is still correct near the force and the singularity of the solution is obtained by the proposed method which is not limited to the computation of points far from the source.

\subsection{Periodic case}

The last example is a truly periodic material made of a network of two materials as seen in figure 14 and plane strain problems are still considered. The 
two materials have the properties $E_{1}=2.10^{10} \mathrm{~Pa}, \nu_{1}=0.3, \rho_{1}=7800 \mathrm{~kg} / \mathrm{m}^{3}$ and $E_{2}=2.10^{9} \mathrm{~Pa}, \nu_{2}=0.3$ and $\rho_{2}=2000 \mathrm{~kg} / \mathrm{m}^{3}$. Each material occupies a square of size $l_{1}=0.025 \mathrm{~m} \times l_{2}=0.025 \mathrm{~m}$, so the periodic cell has the size $L_{1}=0.05 \mathrm{~m} \times L_{2}=0.05 \mathrm{~m}$. The figure 15 presents the comparison between the numerical Green's function and a finite element calculation with a structure made of $40 \times 40$ periods plus 20 layers with increasing dissipation as in the perfectly matched layer method to avoid wave reflections at boundaries. A good agreement between the two solutions is found. Some differences can be seen at low frequencies for which the finite element solution is no more accurate since the size of the absorbing layer becomes insufficient for these frequencies.

The full finite element problem contains 640000 elements and 641601 nodes and a typical cell is meshed with 100 elements and 121 nodes. In the periodic computation a cell is also meshed with 100 elements and 121 nodes, so with the same density as for the full problem. For the numerical Green's function presented in this article, it is necessary to do different computations for different values of the transverse wavenumber $k$. However it is much more efficient to do a large number of computations on a structure with 121 nodes than a single computation on a structure with 641601 nodes. With the parameter used for the two materials the shear velocities are $c_{1}=1601 \mathrm{~m} / \mathrm{s}$ and $c_{2}=620 \mathrm{~m} / \mathrm{s}$. For the maximum frequency the wavelengths are $0.53 \mathrm{~m}$ and $0.21 \mathrm{~m}$ which are much larger than the size of a cell. The mesh density is sufficient at these 
frequencies.

\section{Conclusion}

We have presented a method to calculate numerically the Green's function for homogeneous or periodic media in the two-dimensional case. Good agreements were found in the cases where an analytical solution is known. Very general media could be considered as this method needs only to post process small size finite element models to get the mass and stiffness matrices of a period in the medium.

This process could be extended firstly to increase the numerical efficiency of the Green's function computation mainly if this has to be used in a boundary element formulation. This can be done easily if different points are to be calculated at the same time as the propagation constants and the wave shapes have to be calculated only once for different points. Asymptotic methods like the stationary phase method could also be considered. Finally, the three-dimensional case has to be considered in a future work.

\section{References}

[1] Morse P.M. And Feshbach H., Methods of Theoretical Physics, McGrawHill, New York, 1953. 
[2] Courant D. And Hilbert D. Methods of Mathematical Physics, WileyInterscience Publications, New York, 1953.

[3] Carslaw H.S. and Jaeger J.C., Conduction of Heat in Solids, $2^{\text {nd }}$ Edition, Clarendon Press, Oxford, 1959.

[4] Ronch G.F., Green's functions, $2^{\text {nd }}$ Edition, Cambridge University Press, Cambridge, England, 1982.

[5] Bonnet M., Boundary integral equation method for solids and fluids, Wiley, Chichester, England, 1995.

[6] Diaz-Contreras R.E. And Nomura S., Green's function applied to solution of Mindlin plates, Computers \& Structures, 60, No. 1, 41-48, 1996.

[7] Dravinski M. And Zheng T., Numerical evaluation of three-dimensional time-harmonic Green's functions for a non isotropic full-space, Wave motions, 32, 141-151, 2000.

[8] Phan A.V., Gray L.J. And Kaplan T., Residue approach for evaluating the 3D anisotropic elastic Green's function: multiple roots, Engineering Analysis with Boundary Elements, 29, 570-576, 2005.

[9] Sales M.A. And Gray L.J., Evaluation of the anisotropic Green's function and its derivatives, Computers and Structures, 69, 247-254, 1998.

[10] Tonon F., Pan E. And Amadei B., Green's functions and boundary element method formulation for 3D anisotropic media, Computers and Structures, 79, 469-482, 2001. 
[11] Yang B. AND PAN E., Three-dimensional Green's functions in anisotropic trimaterials, Int. Jour. Sol. Struc., 39, 2235-2255, 2002.

[12] Bai H., Zhu J., Shah A.H. And Popplewell N., Three-dimensional steady state Green function for a layered isotropic plate, J. Sound Vib., 269, 251-271, 2004.

[13] PAN E., Static Green's functions in multilayered half spaces, Appl. Math. Modelling, 21, 509-521, 1997.

[14] SpIEs M., Green's tensor function for Lamb's problem: The general anisotropic case, J. Acous. Soc. Am., 102, 2438-2441, 1997.

[15] He L.H. And Lim C.W., Surface Green function for a soft elastic half-space: Influence of surface stress, Int. Jour. Sol. Struc., 43, 132-143, 2006.

[16] Ding H.J., Chen W.Q. And Jiang A.M., Green's functions and boundary element method for transversely isotropic piezoelectric materials, Engineering Analysis with Boundary Elements, 28, 975-987, 2004.

[17] Mace B.R., Duhamel D., Brennan M.J. and Hinke L., Finite element prediction of wave motion in structural waveguides, J. Acous. Soc. Amer., 117, pp 2835-2843, 2005.

[18] Duhamel D., Mace B.R. And Brennan M.J., Finite element analysis of the vibrations of waveguides and periodic structures, J. Sound Vib., 294, 205-220, 2006.

[19] LANGLEY R.S., The response of two-dimensional periodic structures to point harmonic forcing, J. Sound Vib., 197, pp 447-469, 1996. 
[20] LANGLEY R.S., The response of two-dimensional periodic structures to impulsive point loading, J. Sound Vib., 201, pp 235-253, 1997.

[21] Langley R.S., Bardell N.S. and Ruivo H.M., The response of twodimensional periodic structures to harmonic point loading: a theoretical and experimental study of a beam grillage, J. Sound Vib., 207, pp 521-535, 1997.

[22] Abdel-Rahman A.Y.A., Matrix analysis of wave propagation in periodic systems, PhD thesis, University of Southampton, Southampton, England, 1979.

[23] www.ansys.com

[24] www.mathworks.com

\section{A Decomposition into pseudo periodic solutions}

Consider the structure shown schematically in figure 2 which has periodic mechanical properties and is defined on $R^{2}$. The first step is to decompose functions defined on this structure into a sum of pseudo periodic functions. A point in $R^{2}$ is denoted $\left(x_{1}, x_{2}\right)$. A square integrable function in $R^{2}$ can be decomposed by a partial Fourier transform as

$$
U\left(x_{1}, x_{2}\right)=\frac{1}{2 \pi} \int_{-\infty}^{+\infty} U\left(x_{1}, k\right) e^{i k x_{2}} d k
$$

The integration on $k$ can be further decomposed into a sum of integrals by

$$
U\left(x_{1}, x_{2}\right)=\frac{1}{2 \pi} \sum_{m_{2}=-\infty}^{+\infty} \int_{\left(2 m_{2}-1\right) \frac{\pi}{L_{2}}}^{\left(2 m_{2}+1\right) \frac{\pi}{L_{2}}} U\left(x_{1}, k\right) e^{i k x_{2}} d k
$$


where $L_{2}$ is the spatial period along dimension 2. This can also be written under the form

$$
U\left(x_{1}, x_{2}\right)=\int_{-\frac{\pi}{L_{2}}}^{\frac{\pi}{L_{2}}} u\left(x_{1}, k, x_{2}\right) e^{i k x_{2}} d k
$$

where $u\left(x_{1}, k, x_{2}\right)$ is a periodic function in $x_{2}$ given by

$$
u\left(x_{1}, k, x_{2}\right)=\frac{1}{2 \pi} \sum_{m_{2}=-\infty}^{+\infty} U\left(x_{1}, k+2 \pi \frac{m_{2}}{L_{2}}\right) e^{2 \pi i \frac{m_{2}}{L_{2}} x_{2}}
$$

It can be proved, by decomposing the periodic function $u$ into Fourier series in formula (A-3), that the function $u$ is uniquely determined by $U$.

As an example of decomposition consider a point force at $\left(x_{1}^{s}, x_{2}^{s}\right)$ whose expression is

$$
\mathbf{F}\left(x_{1}, x_{2}\right)=\mathbf{F} \delta\left(x_{1}-x_{1}^{s}\right) \delta\left(x_{2}-x_{2}^{s}\right)
$$

where $\mathbf{F}$ is a vector giving the amplitude and the direction of the force. The partial Fourier transform of this solution is

$$
\mathbf{F}\left(x_{1}, k\right)=\mathbf{F} \delta\left(x_{1}-x_{1}^{s}\right) e^{-i k x_{2}^{s}}
$$

So in formula (A-4), the periodic function of $\mathbf{x}$ is given by

$$
\mathbf{f}\left(x_{1}, k, x_{2}\right)=\frac{1}{2 \pi} \mathbf{F} e^{-i k x_{2}^{s}} \delta\left(x_{1}-x_{1}^{s}\right) \sum_{m_{2}=-\infty}^{+\infty} e^{2 \pi i \frac{m_{2}}{L_{2}}\left(x_{2}-x_{s}^{2}\right)}
$$


It is known that the sum can be written as a periodic sum of delta functions (consider the Fourier series of the delta function). This leads to the periodic sum

$$
\mathbf{f}\left(x_{1}, k, x_{2}\right)=\frac{L_{2}}{2 \pi} \mathbf{F} e^{-i k x_{2}^{s}} \delta\left(x_{1}-x_{1}^{s}\right) \sum_{m_{2}=-\infty}^{+\infty} \delta\left(x_{2}-x_{2}^{s}-m_{2} L_{2}\right)
$$

Taking the restriction of this function to a period gives

$$
\mathbf{f}\left(x_{1}, k, x_{2}\right)_{\text {period }}=\frac{L_{2}}{2 \pi} \mathbf{F} e^{-i k x_{2}^{s}} \delta\left(x_{1}-x_{1}^{s}\right) \delta\left(x_{2}-x_{2}^{p}\right)
$$

where $x_{2}^{p}$ is the number in the sequel $x_{2}^{s}+m_{2} L_{2}$ which is in the considered cell. 


\section{List of Figures}

$1 \quad$ Green's function. 35

$\begin{array}{lll}2 & \text { Periodic medium. } & 36\end{array}$

3 Transverse and longitudinal degrees of freedom. 37

$4 \quad$ Reduced set of degrees of freedom.

$\begin{array}{lll}5 & \text { A quadrilateral cell. } & 39\end{array}$

$\begin{array}{lll}6 & \text { Four nodes acoustic element. } & 40\end{array}$

7 Comparison of analytical and numerical Green's functions for the acoustic case: $\quad$ real part analytical,.-- . imaginary part analytical, * real part numerical, o imaginary part numerical. $\quad 41$

$8 \quad$ Error in the Green's function versus the number of elements in \begin{tabular}{|c|c|}
\hline & \\
\hline the periodic cell for the acoustic case: & $1 \mathrm{x} 1 \mathrm{mesh}, \bullet 2 \mathrm{x} 2 \mathrm{mesh}$ \\
\hline
\end{tabular} .$--.4 \mathrm{x} 4$ mesh.

$9 \quad$ Error in the Green's function versus the size of the periodic cell for the acoustic case: $\mathrm{a}=\mathrm{b}=0.05 \mathrm{~m}, \bullet \mathrm{a}=\mathrm{b}=0.01 \mathrm{~m},-.-$. $\mathrm{a}=\mathrm{b}=0.005 \mathrm{~m}, * \mathrm{a}=\mathrm{b}=0.001 \mathrm{~m}$, o $\mathrm{a}=\mathrm{b}=0.0005 \mathrm{~m}$. 
10 Comparison of analytical and numerical Green's functions for the two-dimensional elasticity: $\quad$ real part analytical,.-- . imaginary part analytical, * real part numerical, o imaginary part numerical.

11 Error in the Green's function versus the number of elements in the periodic cell for the two-dimensional elasticity: 1 1x1 mesh. - 2x2 mesh, -. -. .4x4 mesh, o 1x1 mesh of degree 2.

12 Error in the Green's function versus the size of the periodic \begin{tabular}{l|l} 
& \\
cell for the two-dimensional elasticity: & $L_{1}=L_{2}=0.05 \mathrm{~m}$, \\
\hline
\end{tabular} - $L_{1}=L_{2}=0.02 m,-.-. L_{1}=L_{2}=0.01 m, \times$ $L_{1}=L_{2}=0.005 m$

13 Comparison of analytical and numerical Green's functions for \begin{tabular}{l|l|} 
& \\
the two-dimensional elasticity: & real part analytical,.-- \\
\hline
\end{tabular} imaginary part analytical, x real part numerical, o imaginary part numerical. 
15 Comparison of the present method and a full FEM computation

for a periodic two materials medium: a) $x$ component of

\begin{tabular}{|c|c|c|}
\hline displacement at point $(0.25,0)$, b) $y$ component of displacement \\
\hline
\end{tabular}

at point $(0.25,0), \mathrm{c}) x$ component of displacement at point

$(0.5,0.5)$, d) $y$ component of displacement at point $(0.5,0.5)$,

\begin{tabular}{|l|l|}
\hline & \\
\hline & real part present method,.-- . . imaginary part present \\
\hline
\end{tabular}

method, o real part full FEM, + imaginary part full FEM. 


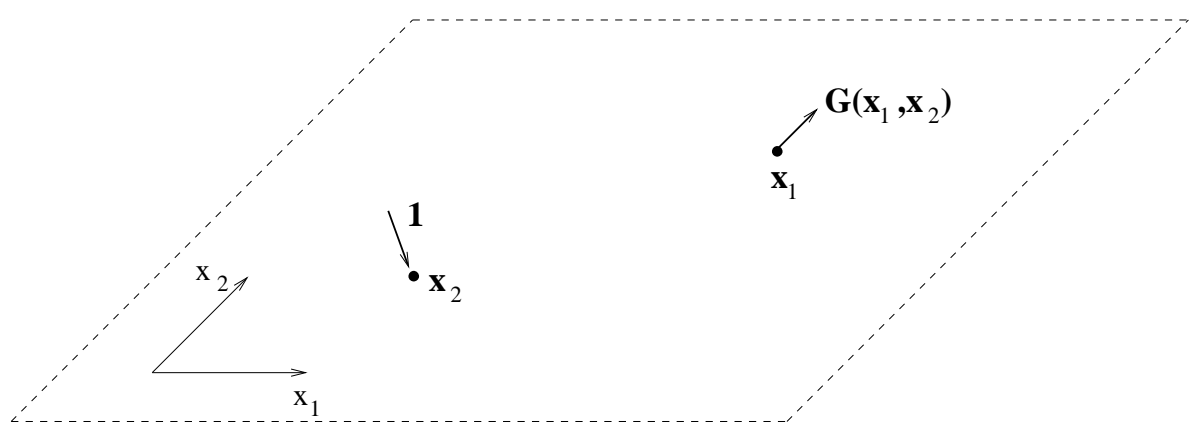

Fig. 1. Green's function. 


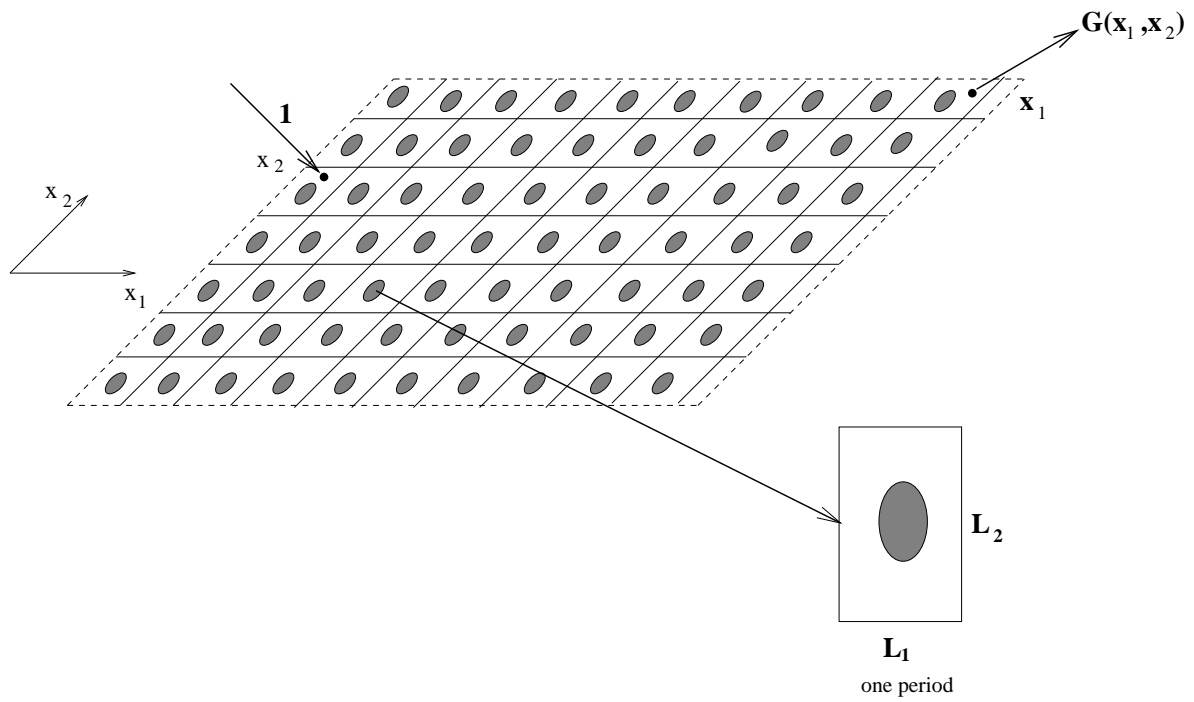

Fig. 2. Periodic medium. 


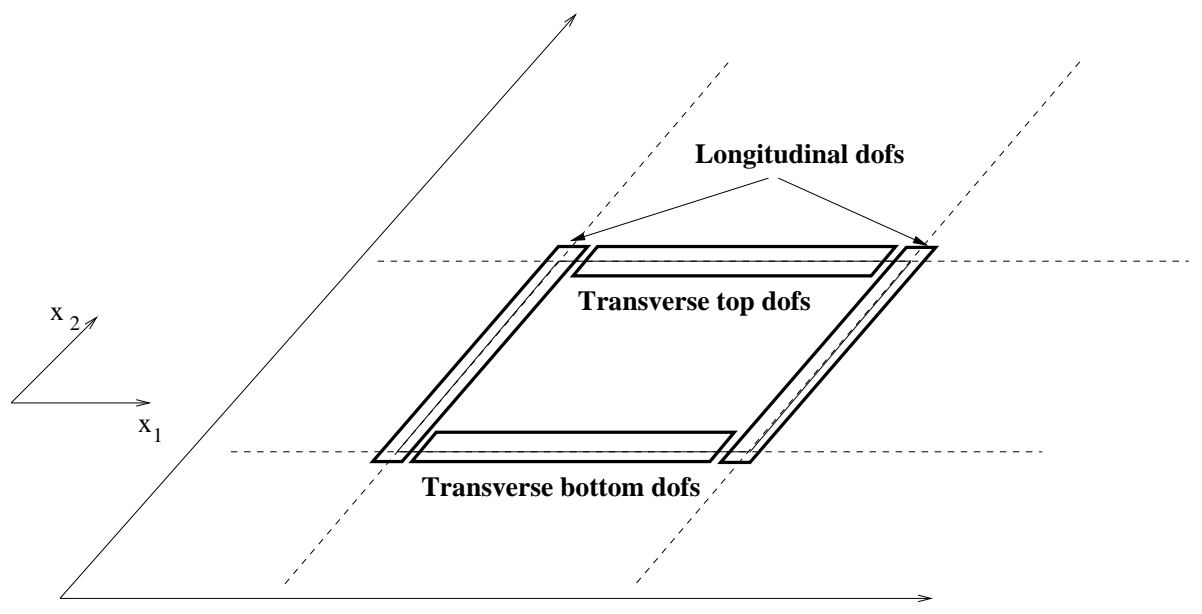

Fig. 3. Transverse and longitudinal degrees of freedom. 


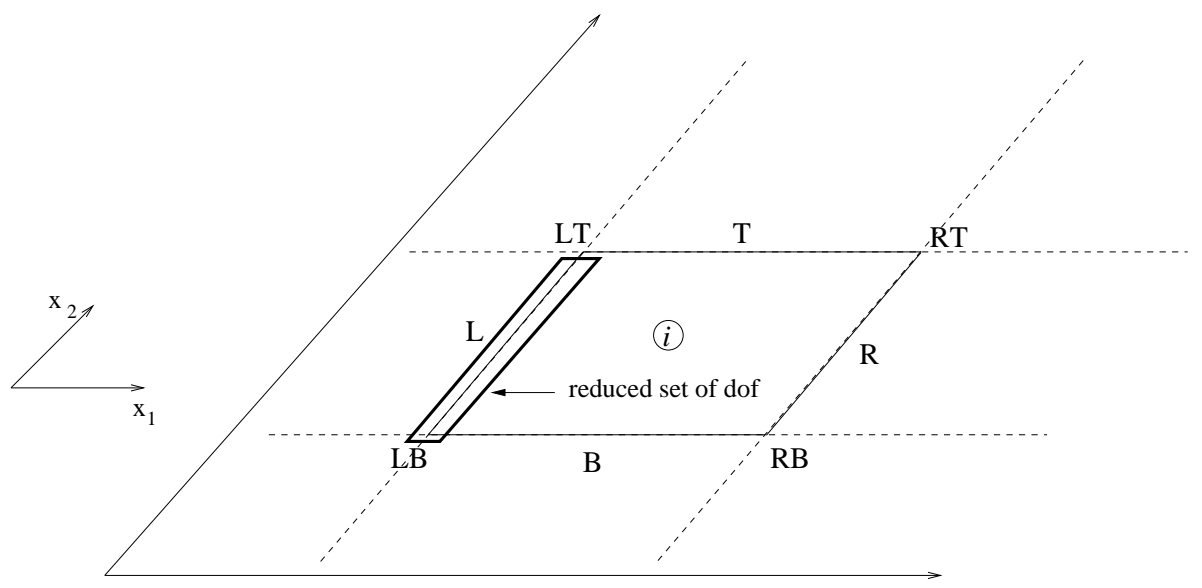

Fig. 4. Reduced set of degrees of freedom. 


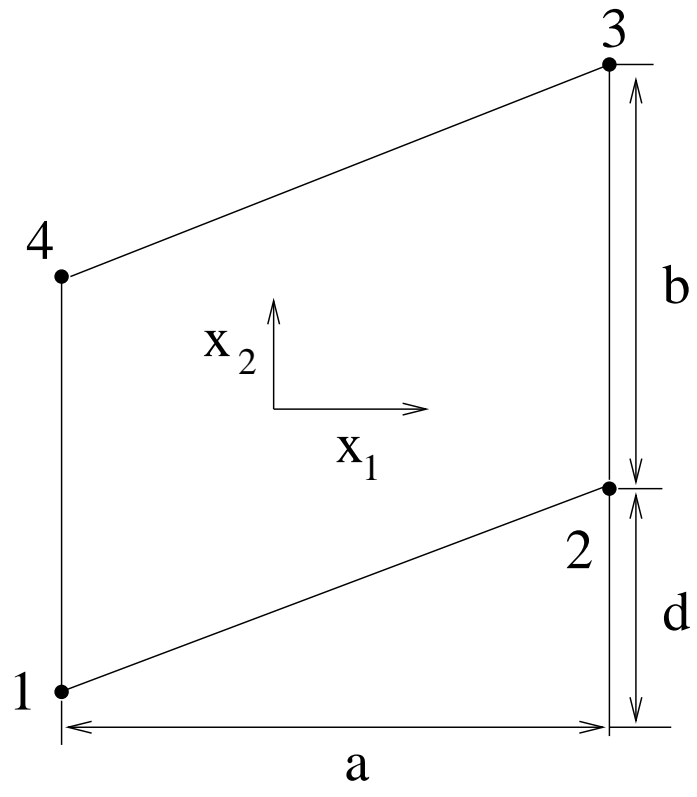

Fig. 5. A quadrilateral cell. 


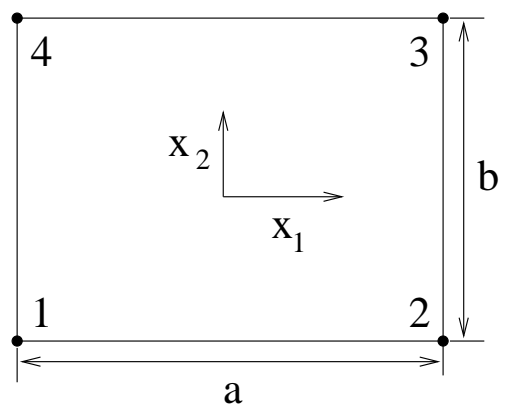

Fig. 6. Four nodes acoustic element. 


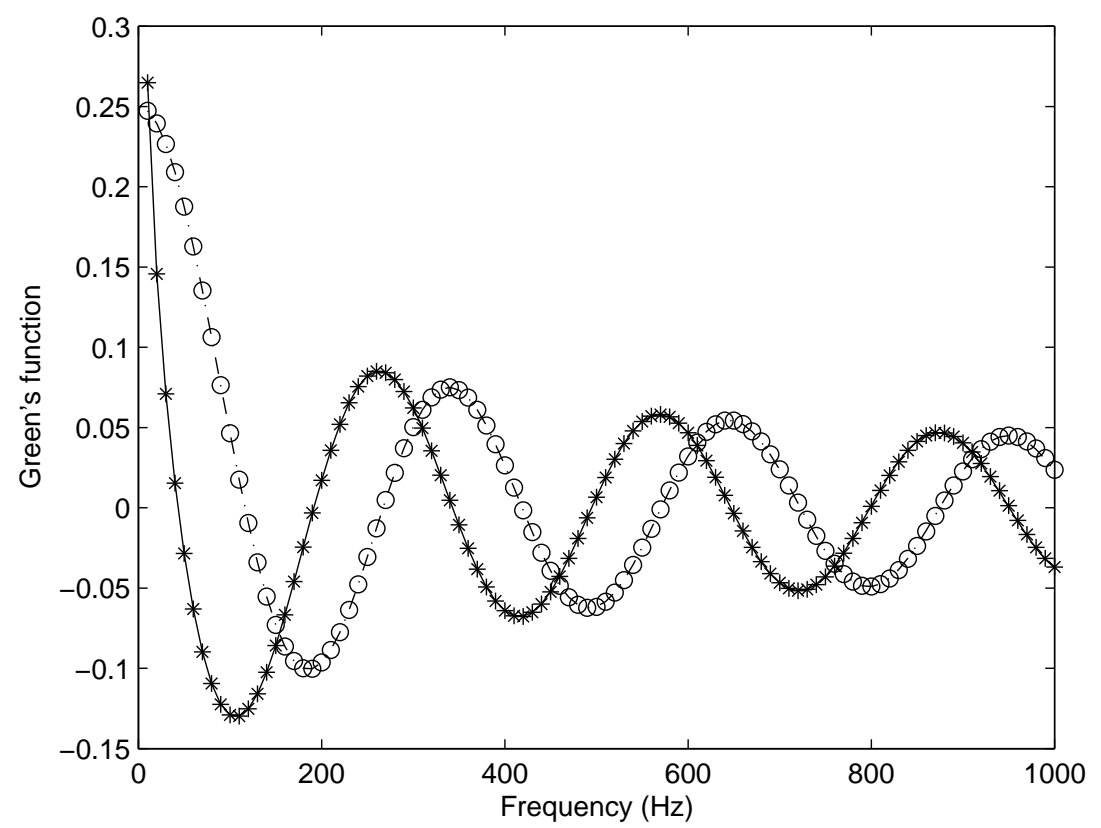

Fig. 7. Comparison of analytical and numerical Green's functions for the acoustic case: real part analytical, -.-. imaginary part analytical, * real part numerical, o imaginary part numerical. 


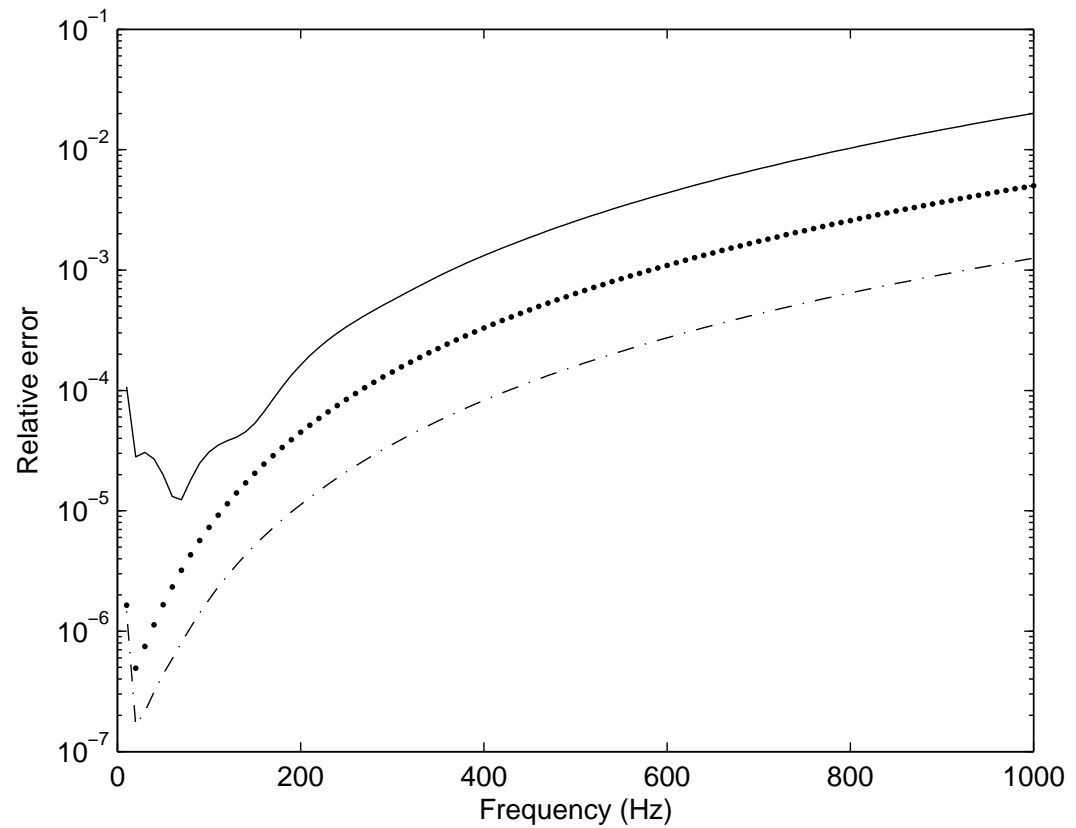

Fig. 8. Error in the Green's function versus the number of elements in the periodic cell for the acoustic case: __ 1x1 mesh, • 2x2 mesh, _. - . 4 x4 mesh. 


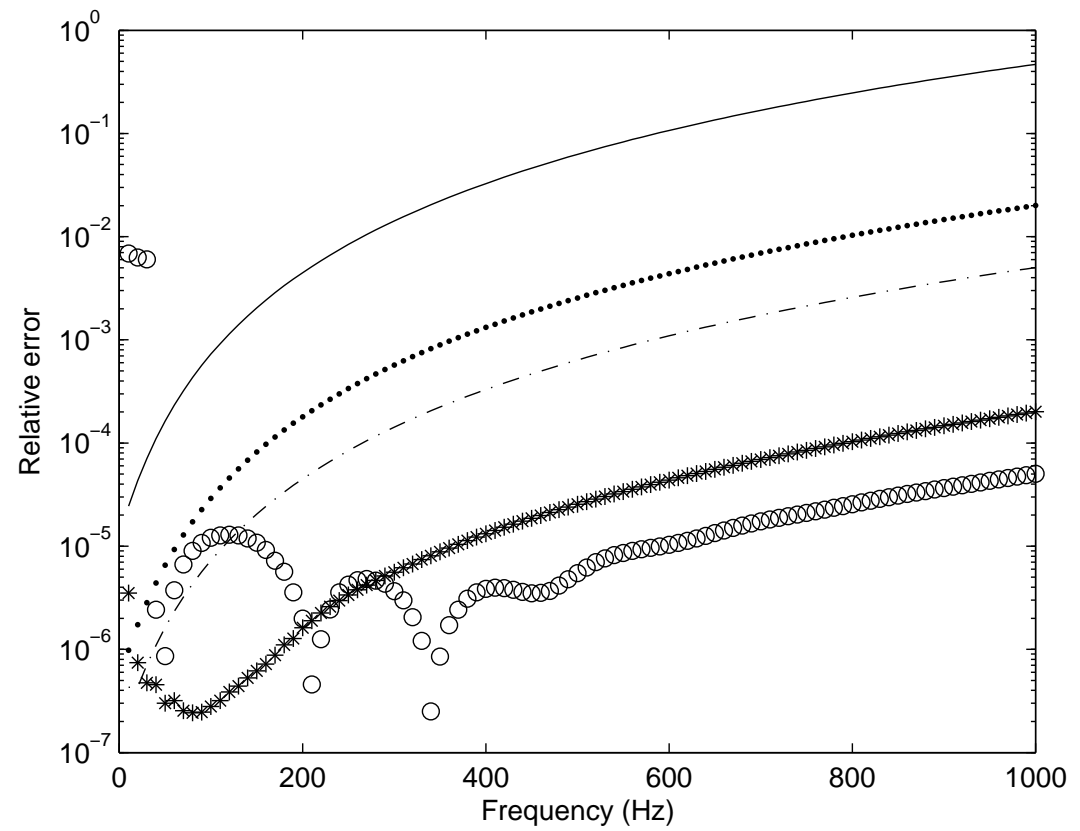

Fig. 9. Error in the Green's function versus the size of the periodic cell for the acoustic case: $\mathrm{a}=\mathrm{b}=0.05 \mathrm{~m}, \bullet \mathrm{a}=\mathrm{b}=0.01 \mathrm{~m},-.-. \mathrm{a}=\mathrm{b}=0.005 \mathrm{~m}, * \mathrm{a}=\mathrm{b}=0.001 \mathrm{~m}$, $\mathrm{o} a=b=0.0005 \mathrm{~m}$. 


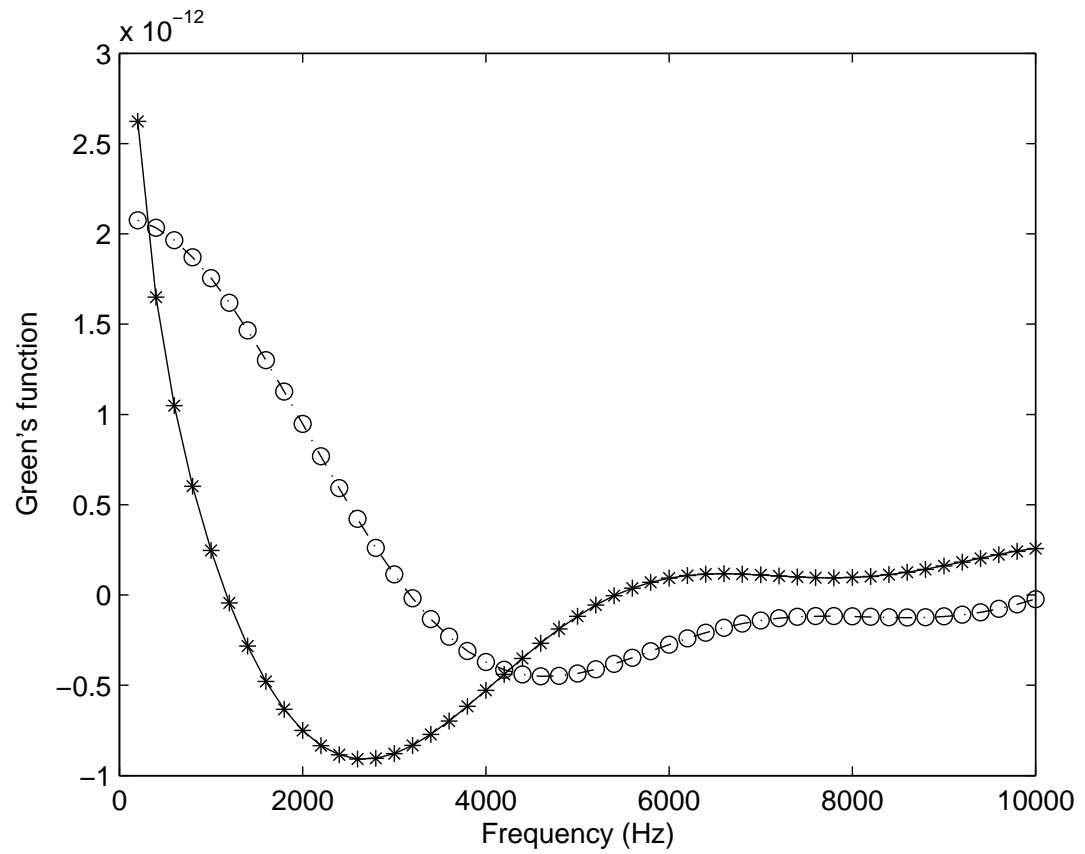

Fig. 10. Comparison of analytical and numerical Green's functions for the two-dimensional elasticity: __ real part analytical, _. - . imaginary part analytical, * real part numerical, o imaginary part numerical. 


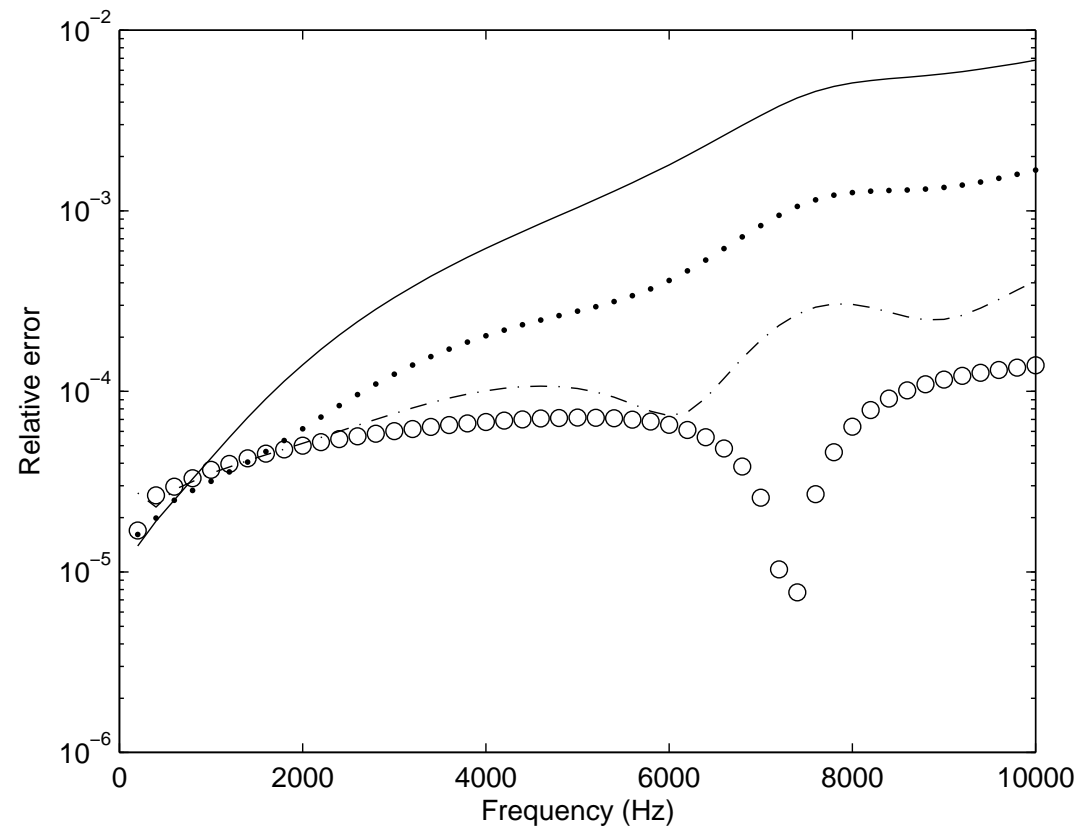

Fig. 11. Error in the Green's function versus the number of elements in the periodic cell for the two-dimensional elasticity: 1x1 mesh, • 2x2 mesh, -. - . $4 \times 4$ mesh, o $1 x 1$ mesh of degree 2 . 


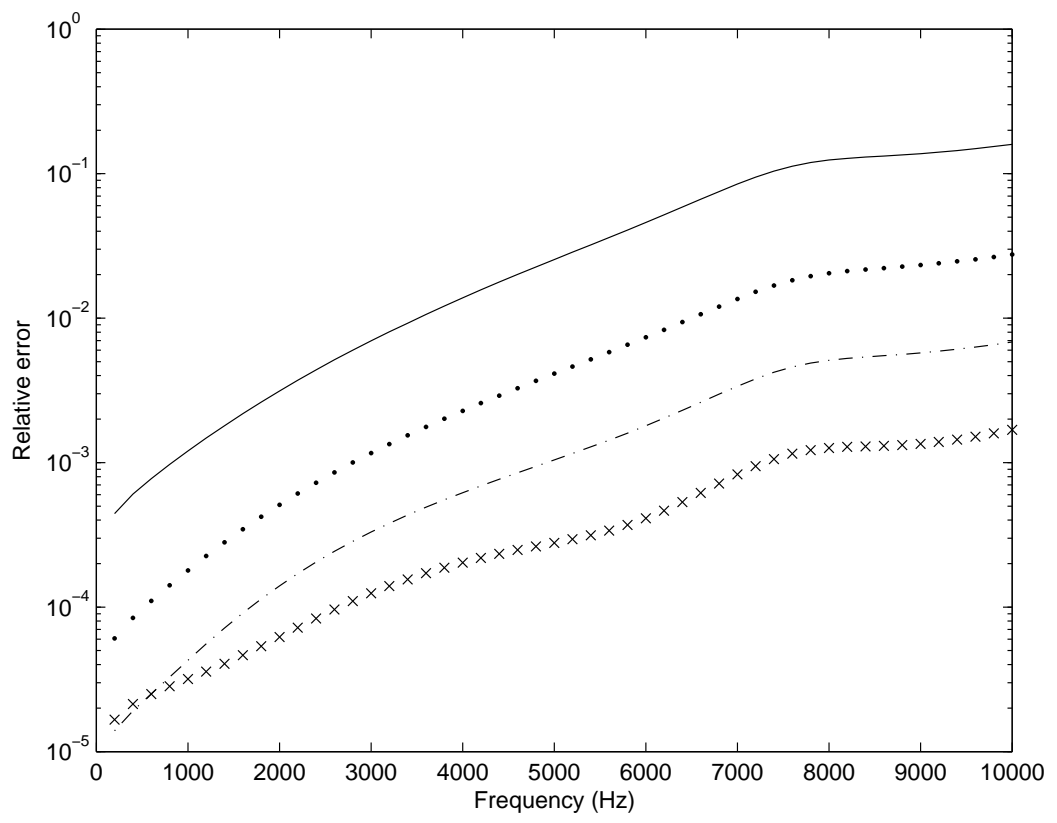

Fig. 12. Error in the Green's function versus the size of the periodic cell for the two-dimensional elasticity: $\_L_{1}=L_{2}=0.05 m, \bullet L_{1}=L_{2}=0.02 m,-$. - . $L_{1}=L_{2}=0.01 \mathrm{~m}, \times L_{1}=L_{2}=0.005 \mathrm{~m}$. 


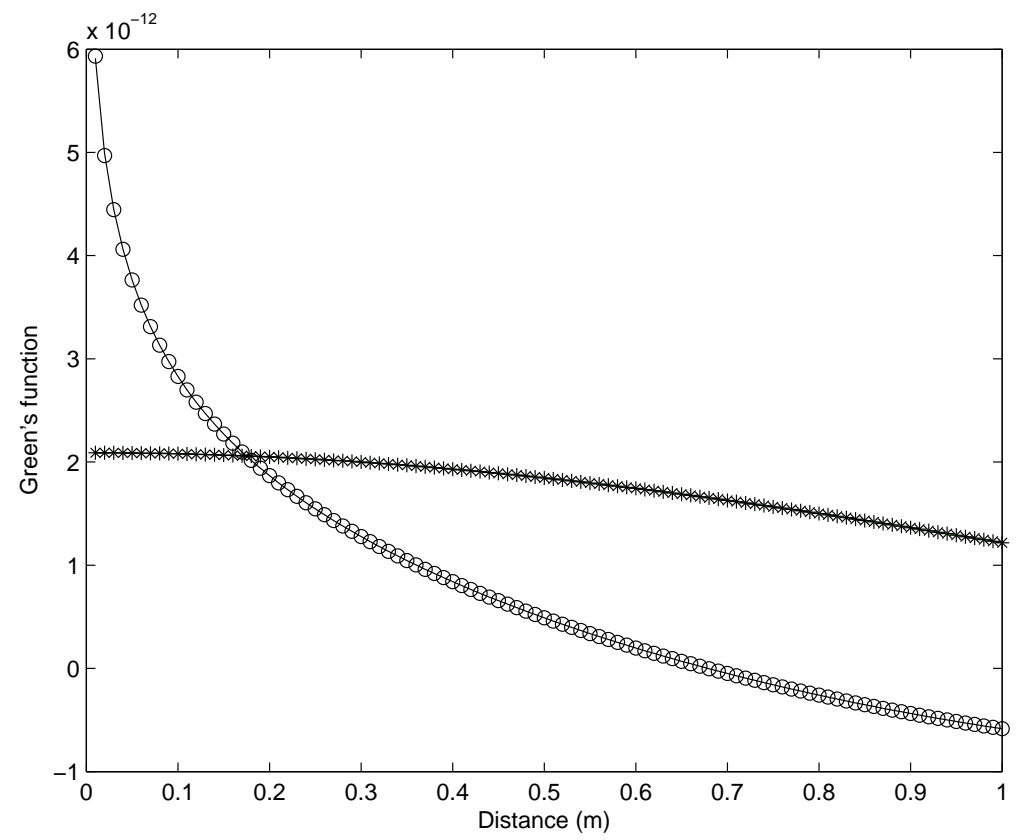

Fig. 13. Comparison of analytical and numerical Green's functions for the two-dimensional elasticity: real part analytical, -. - . imaginary part analytical, x real part numerical, o imaginary part numerical. 


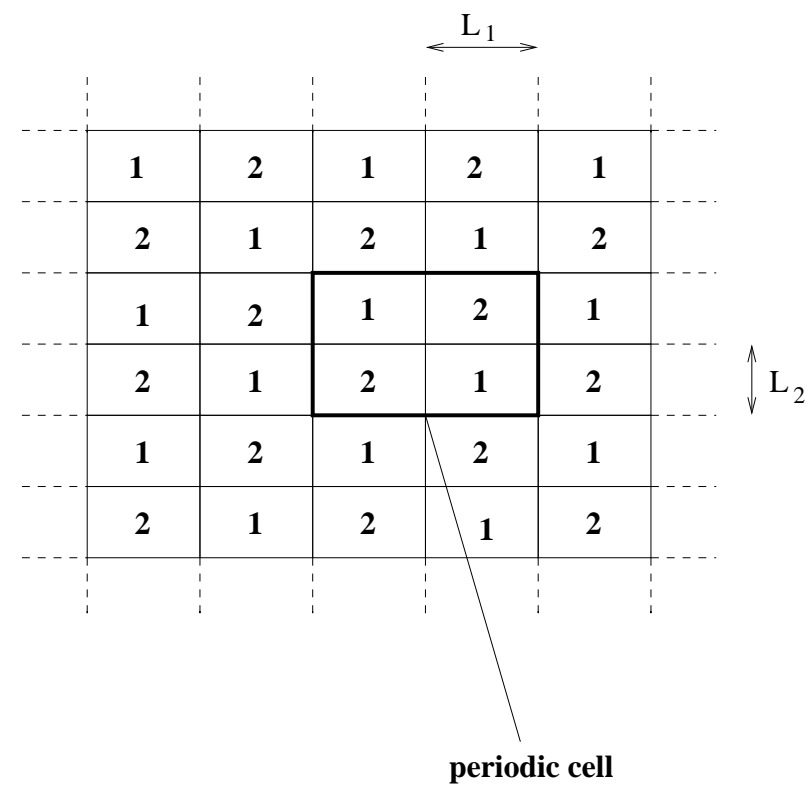

Fig. 14. Network of two materials. 

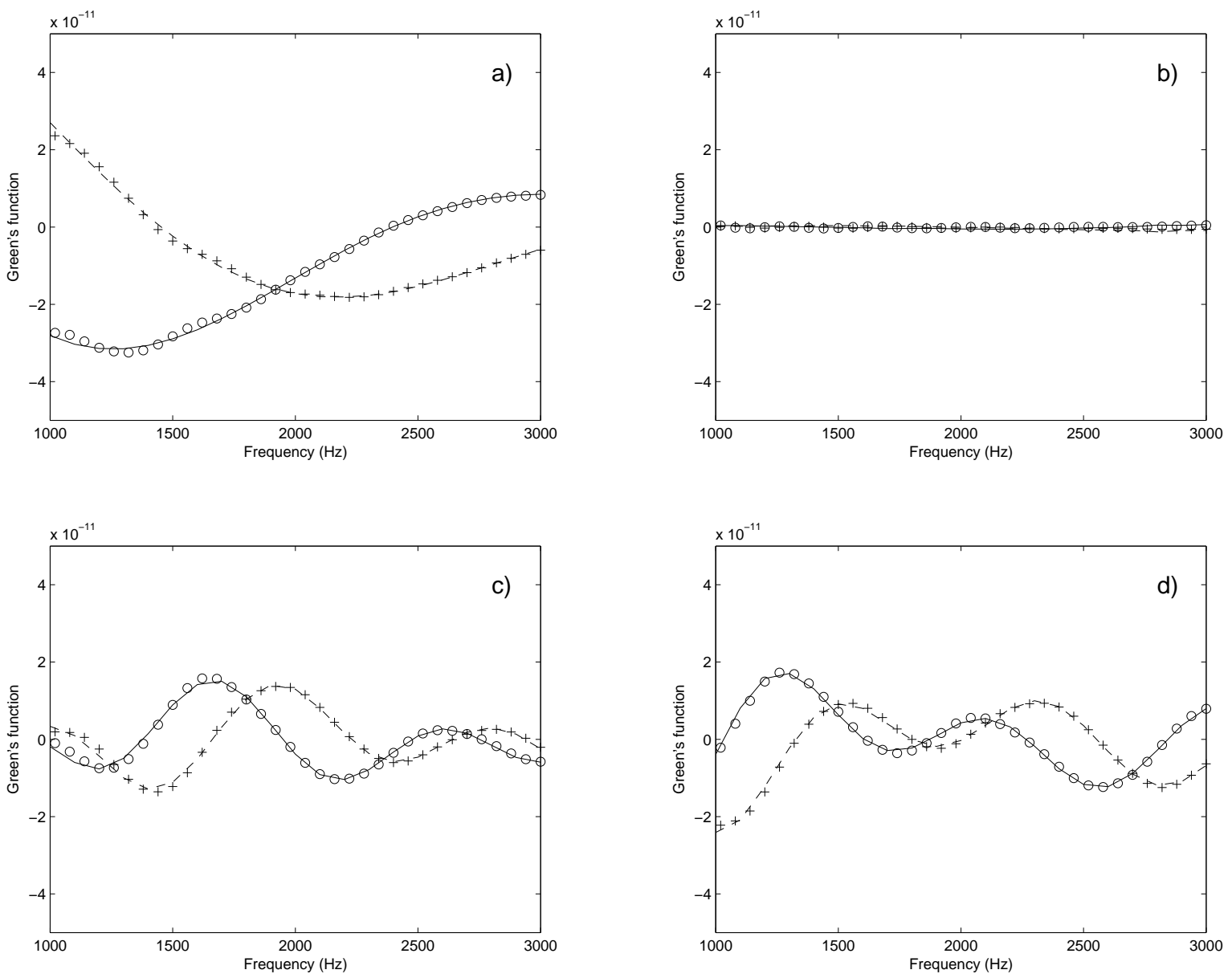

Fig. 15. Comparison of the present method and a full FEM computation for a periodic two materials medium: a) $x$ component of displacement at point $(0.25,0)$, b) $y$ component of displacement at point $(0.25,0)$, c) $x$ component of displacement at point $(0.5,0.5), \mathrm{d}) y$ component of displacement at point $(0.5,0.5)$, real part present method, -. - . imaginary part present method, o real part full FEM, + imaginary part full FEM. 